\title{
Value of the resting 12 lead electrocardiogram and vectorcardiogram for locating the accessory pathway in patients with the Wolff-Parkinson-White syndrome
}

\author{
ROBERT LEMERY, „ STEPHEN C HAMMILL, * DOUGLAS L WOOD, * \\ GORDON K DANIELSON, $\dagger$ HAROLD T MANKIN, MICHAEL J OSBORN, \\ BERNARD J GERSH, * DAVID R HOLMES JR* \\ From the ${ }^{\star}$ Division of Cardiovascular Diseases and Internal Medicine and the $\dagger$ Section of Thoracic and \\ Cardiovascular Surgery, Mayo Clinic and Mayo Foundation, Rochester, Minnesota, USA
}

SUMMARY The resting 12 lead electrocardiogram and vectorcardiogram were reviewed in 47 patients with the Wolff-Parkinson-White syndrome $(a)$ who had pre-excitation on the resting 12 lead electrocardiogram, $(b)$ who had a single anterograde conducting accessory pathway assessed and located during preoperative electrophysiological study and during epicardial mapping at operation, and $(c)$ in whom surgical division of the accessory pathway resulted in loss of preexcitation. The site of the accessory pathway established during operation was compared with that established by evaluating the polarity of the delta wave and QRS complex on the resting 12 lead electrocardiogram. The electrocardiogram was assessed by the Rosenbaum criteria (Wolff-Parkinson-White type A, left-sided pathway; or type B, right-sided pathway), the Gallagher criteria (atrial pacing resulting in maximal pre-excitation), and the World Health Organisation criteria (a composite of previous studies). The Gallagher and World Health Organisation criteria were derived from patients demonstrating maximal pre-excitation that often required atrial pacing. The present study was designed to determine whether these criteria could be accurately applied to the resting 12 lead electrocardiogram on which the degree of pre-excitation was variable. The Rosenbaum criteria correctly identified a left sided accessory pathway in 26 of 34 patients and a right-sided accessory pathway in nine of 13 patients. The Gallagher and World Health Organisation criteria correctly identified the location in only 15 $(32 \%)$ of the 47 patients. The resting vectorcardiogram was inaccurate for locating the accessory pathway.

Although published criteria are useful for identifying the site of the accessory pathway from an electrocardiogram obtained when rapid atrial pacing is being used to achieve maximal pre-excitation, they are not suitable for identifying the exact site of an accessory pathway from the resting 12 lead electrocardiogram.

The electrocardiogram from patients with the Wolff-Parkinson-White syndrome ${ }^{1-3}$ classically demonstrates a short PR interval, a broad QRS complex, and typical slurring of the initial portion of the

Requests for reprints to Dr Stephen C Hammill, Mayo Clinic, 200 First Street SW, Rochester, Minnesota 55905, USA.

Accepted for publication 12 May 1987
QRS complex, referred to as a delta wave. ${ }^{4}$ The $P R$ interval and the width of the QRS complex, or degree of pre-excitation, may vary ${ }^{23}$ depending on the location of the accessory pathway; the intraatrial, atrioventricular node, His-Purkinje, and accessory pathway conduction times; autonomic tone; and cardioactive medications. ${ }^{235}$ These factors may result in a spectrum of findings, including no apparent pre-excitation, varying degrees of 
pre-excitation, or maximal pre-excitation, on the resting 12 lead electrocardiogram in patients with the Wolff-Parkinson-White syndrome.

Before electrophysiological testing, knowledge of the location of an accessory pathway is useful because it may be important initial evidence of multiple accessory pathways, especially in patients who have one pathway that conducts only in the anterograde direction and accounts for the delta wave and a second pathway that conducts only in the retrograde direction and participates in reentrant tachycardia (concealed accessory pathway). ${ }^{6}$ Criteria for locating the accessory pathway from the 12 lead electrocardiogram have been published. $^{1-3}$ These criteria ${ }^{23}$ were derived from electrocardiograms demonstrating maximal pre-excitation. This often requires rapid atrial pacing. The use of such criteria to determine accurately the location of the accessory pathway from the resting electrocardiogram has not previously been assessed and forms the basis for this study.

\section{Patients and methods}

\section{PATIENTS}

Between 1976 and 1985, 47 patients (32 male and 15 female) with the Wolff-Parkinson-White syndrome met the following criteria for entry into this study: (a) presence of a delta wave on the resting 12 lead electrocardiogram; (b) presence of a single anterograde conducting accessory pathway found at preoperative electrophysiological study and confirmed by epicardial mapping during operation; and $(c)$ loss of pre-excitation after surgical division of the accessory pathway. The mean age of the patients was 28 years (range 7-65 years). Eight patients had associated cardiac disorders: dilated cardiomyopathy and left lateral accessory pathway in one, atrial septal defect and left paraseptal accessory pathway in one, and Ebstein's anomaly with a right-sided accessory pathway in six. Four patients had hypertension.

\section{ELECTROCARDIOGRAM}

The resting 12 lead electrocardiogram was recorded at $25 \mathrm{~mm} / \mathrm{s}$ and a gain of $10 \mathrm{~mm} / \mathrm{mV}$. All patients were in sinus rhythm, and the mean heart rate was 71 beats/min (range $47-113$ beats/min). If multiple electrocardiograms were obtained from a patient, the one demonstrating the most pre-excitation was chosen for analysis. All recordings were analysed by two of us. The delta wave polarity was recorded as being positive, negative, or isoelectrical during the first $40 \mathrm{~ms}$ of the beginning of the QRS complex (positive, most or all of the initial slurring with a positive deflection; negative, most or all of the initial slurring with a negative deflection; and isoelectrical, no slurring of the initial part of the QRS or an initial downward or upward deflection followed by a reversed deflection to the baseline during the first $40 \mathrm{~ms}$ of the QRS complex).

In 32 patients, three simultaneous channels of the electrocardiogram were recorded. These were grouped as follows: I, II, and III; aVR, aVL, and aVF; V1, V2, and V3; and V4, V5, and V6. In these patients the onset of the delta wave was determined as the time when the initial deflection was observed in one of the three simultaneously recorded leads. In the 15 remaining patients, the recordings were not simultaneous, and the delta wave was analysed independently in each lead.

\section{VECTORCARDIOGRAM}

The vectorcardiogram was obtained simultaneously with the 12 lead electrocardiogram in each patient. Computer analysis allowed for determination of the vector direction at $10,20,30$, and $40 \mathrm{~ms}$, with a mean vector at $30 \mathrm{~ms}(\mathrm{R} 30)$.

\section{ELECTROPHYSIOLOGICAL EVALUATION AND EPICARDIAL MAPPING}

Each patient had electrophysiological testing with methods that have previously been reported. ${ }^{78}$ Left-sided accessory pathways were located by means of recordings of left atrial and left ventricular electrograms from an electrode catheter placed in the coronary sinus, and right-sided accessory pathways were located by a mapping electrode catheter during ventricular pacing and reciprocating tachycardia. ${ }^{9}$ Epicardial mapping was performed at the time of operation ${ }^{8-10}$ in all patients. The ventricular epicardial activation sequences were recorded dur-

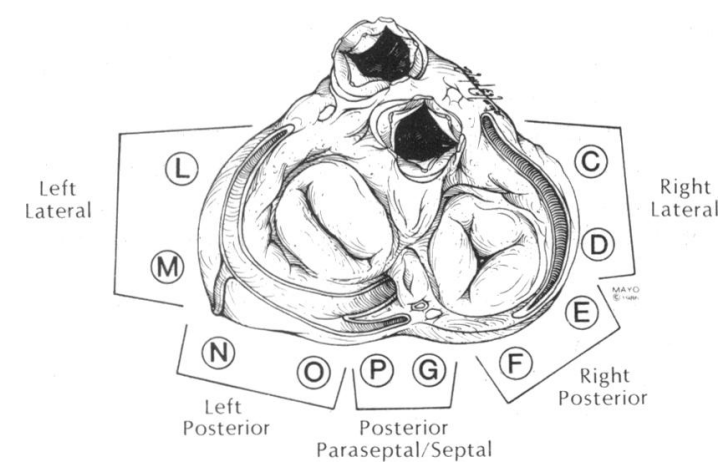

Fig 1 Schematic drawing at the level of the mitral and tricuspid valves, demonstrating epicardial locations during intraoperative electrophysiological mapping $(C-G, L-P)$ to locate accessory atrioventricular pathway. (By permission of the Mayo Foundation.) 
Table 1 World Health Organisation task force criteria for locating the accessory atrioventricular pathway by the resting 12 lead electrocardiogram

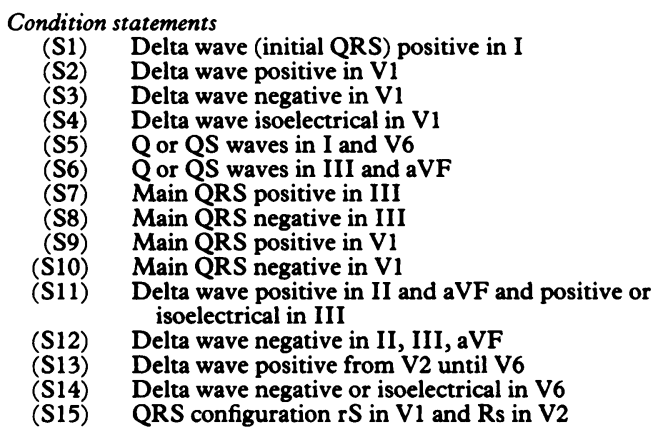

Criterion for anterior right ventricular pre-excitation:

$S 1$ and S4 and S8 and S10

Criterion for posterior right ventricular pre-excitation:

S3 and S6 and S8 and S10

Criterion for posterior left ventricular pre-excitation:

$S 1$ and $S 2$ and $S 6$ and $S 8$ and S9 and S14

Criterion for lateral left ventricular pre-excitation:

S2 and S5 and S7 and S14

Criterion for anterior paraseptal pre-excitation:

$S 1$ and S4 and S11

Criterion for posterior paraseptal pre-excitation:

$S 1$ and $S 12$ and $S 13$ and S15

From Willems et $a^{3}$ by permission of the American College of Cardiology.

ing sinus rhythm and atrial pacing from a site close to the accessory pathway. Atrial activation was determined during ventricular pacing and reciprocating tachycardia in all patients. The site of the accessory pathway was classified as: left lateral, left posterior, posterior paraseptal/septal, right posterior, and right lateral (fig 1).

The surgical approach to division of the accessory pathway has been described elsewhere. ${ }^{810}$ In all patients division of the accessory pathway at the site corresponding to the earliest epicardial activation resulted in disappearance of pre-excitation. This was demonstrated by the surface electrocardiogram, the absence of pre-excitation during incremental atrial pacing, and the presence of type 1 second degree atrioventricular block during atrial pacing.

\section{PUBLISHED CRITERIA FOR LOCATING THE}

\section{ACCESSORY PATHWAY}

In all patients the preoperative resting electrocardiogram was compared with the following criteria: $(a)$ Rosenbaum criteria ${ }^{1}$ (Wolff-Parkinson-White type $A$, in which there is a predominantly positive $Q R S$ complex in V1 and V2, and Wolff-Parkinson-White type $B$, in which there is a predominantly negative QRS complex in V1 and V2; (b) Gallagher criteria ${ }^{2}$ (delta wave polarity, as recorded during rapid atrial

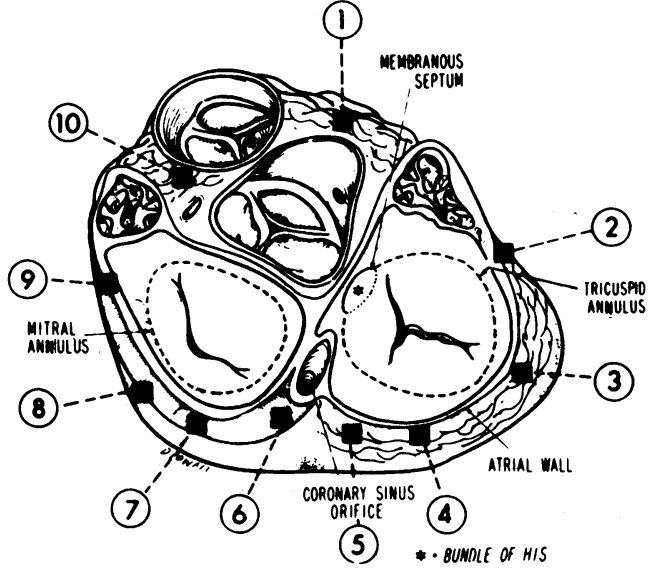

$\begin{array}{ll}\text { 1. RIGHT ANTERIOR PARASEPTAL } & \text { 6. LEFT POSTERIOR PARASEPTAL } \\ \text { 2. RIGHT ANTERIOR } & \text { 7. LEFT POSTERIOR } \\ \text { 3. RIGHT LATERAL } & \text { 8. LEFT LATERAL } \\ \text { 4. RIGHT POSTERIOR } & \text { 9. LEFT ANTERIOR } \\ \text { 5. RIGHT PARASEPTAL } & \text { 10. LEFT ANTERIOR PARASEPTAL }\end{array}$

DELTA waVe pOLARITY

\begin{tabular}{|c|c|c|c|c|c|c|c|c|c|c|c|c|}
\hline & I & II & III & AVR & An & AVF & $v_{1}$ & $v_{2}$ & $v_{3}$ & $v_{4}$ & vs & $v_{0}$ \\
\hline (1) & + & + & $+( \pm)$ & - & $\pm(t)$ & + & \pm & \pm & $+( \pm)$ & + & + & + \\
\hline (2) & + & + & $-\left(\begin{array}{l}+ \\
-1\end{array}\right.$ & - & $+\left(\begin{array}{l}+ \\
+\end{array}\right.$ & $\pm(-)$ & \pm & $+( \pm)$ & $\mid+(+)$ & + & + & + \\
\hline ( & + & $\pm(-)$ & - & - & + & $-\left(t_{-}^{+}\right)$ & \pm & \pm & \pm & + & + & + \\
\hline (a) & + & - & - & - & + & - & $\pm(t)$ & \pm & + & + & + & + \\
\hline (5) & + & - & - & $-(t)$ & + & - & \pm & + & + & + & + & + \\
\hline () & + & - & - & - & + & - & + & + & + & + & + & + \\
\hline (7) & + & - & - & $\pm(t)$ & + & - & + & + & + & + & + & $-( \pm)$ \\
\hline ( ) & $-( \pm)$ & \pm & \pm & $\pm(t)$ & $-( \pm)$ & \pm & + & + & + & + & $-( \pm)$ & $-( \pm)$ \\
\hline (1) & $-( \pm)$ & + & + & - & $-( \pm)$ & + & + & + & + & + & + & + \\
\hline (1) & + & + & $+( \pm)$ & - & \pm & + & $\pm(t)$ & + & + & + & + & + \\
\hline
\end{tabular}

Fig 2 Electrocardiographic classification of Wolff-Parkinson-White syndrome as defined by Gallagher et al. ${ }^{2}$ Ten representative sites are depicted on a cross section of ventricles at level of atrioventricular rings. Expected polarity of delta waves resulting from pre-excitation at these sites is indicated for each of the 12 standard leads, based on analysis of mean initial forces ( $40 \mathrm{~ms}$ ) of ventricular depolarisation. (From Gallagher et al. ${ }^{2}$ By permission of Grune and Stratton.)

pacing resulting in maximal pre-excitation during the electrophysiological study (fig 2)); and (c) World Health Organisation criteria, ${ }^{3}$ which are a composite of previous criteria (table 1 ). We did not require complete concordance between all of the criteria for identification of the site of a pathway: with the Gal- 
ble 2 Delta wave polarity on the resting 12 lead electrocardiogram in patients with left lateral or left posterior accessory pathways

\begin{tabular}{|c|c|c|c|c|c|c|c|c|c|c|c|c|c|c|c|c|}
\hline \multirow[b]{2}{*}{ :essory pathway location } & \multirow[b]{2}{*}{ Patient } & \multicolumn{10}{|c|}{ Lead* } & \multicolumn{2}{|c|}{. } & \multirow{2}{*}{$\begin{array}{l}\text { Type of } \\
W P W+^{1}\end{array}$} & \multicolumn{2}{|c|}{$\begin{array}{l}\text { Concordance of } \\
\text { criteriat }\end{array}$} \\
\hline & & $I$ & $I I$ & $I I I$ & $\boldsymbol{R}$ & $L$ & $F$ & $V 1$ & $V 2$ & V3 & $V 4$ & $V 5$ & V6 & & Gallagher $^{2}$ & $\mathrm{WHO}^{3}$ \\
\hline t lateral, QRS > $120 \mathrm{~ms}$ & $\begin{array}{l}1 \\
2 \\
3 \\
4 \\
5 \\
6 \\
7 \\
8\end{array}$ & $\begin{array}{l}+ \\
\pm \\
+ \\
\pm \\
\pm \\
+ \\
+ \\
-\end{array}$ & $\begin{array}{l} \pm \\
+ \\
- \\
+ \\
+ \\
+ \\
\pm \\
+\end{array}$ & $\begin{array}{l} \pm \\
+ \\
- \\
+ \\
+ \\
+ \\
\pm \\
+\end{array}$ & $\begin{array}{l} \pm \\
\pm \\
- \\
- \\
- \\
- \\
- \\
\pm\end{array}$ & $\begin{array}{l} \pm \\
\pm \\
\pm \\
\frac{ \pm}{ \pm} \\
\frac{ \pm}{ \pm} \\
-\end{array}$ & $\begin{array}{l} \pm \\
+ \\
- \\
+ \\
+ \\
+ \\
\pm \\
+\end{array}$ & $\begin{array}{l}+ \\
+ \\
+ \\
+ \\
+ \\
+ \\
+ \\
+\end{array}$ & $\begin{array}{l}+ \\
+ \\
+ \\
+ \\
+ \\
+ \\
+ \\
+ \\
+\end{array}$ & $\begin{array}{l}+ \\
+ \\
+ \\
+ \\
+ \\
+ \\
+ \\
+\end{array}$ & $\begin{array}{l}+ \\
+ \\
+ \\
+ \\
+ \\
+ \\
+ \\
+\end{array}$ & $\begin{array}{l}+ \\
+ \\
+ \\
+ \\
+ \\
+ \\
+ \\
+\end{array}$ & $\begin{array}{l} \pm \\
\pm \\
\pm \\
+ \\
+ \\
+ \\
\pm \\
\pm\end{array}$ & $\begin{array}{l}\mathbf{A} \\
\mathbf{A} \\
\mathbf{A} \\
\mathbf{B} \\
\mathbf{A} \\
\mathbf{B} \\
\mathbf{A} \\
\mathbf{A}\end{array}$ & $\begin{array}{l}\mathbf{Y} \\
\mathbf{N} \\
\mathbf{N} \\
\mathbf{N} \\
\mathbf{N} \\
\mathbf{N} \\
\mathbf{Y} \\
\mathbf{N}\end{array}$ & $\begin{array}{l}\mathbf{N} \\
\mathbf{Y} \\
\mathbf{N} \\
\mathbf{N} \\
\mathbf{N} \\
\mathbf{N} \\
\mathbf{N} \\
\mathbf{Y}\end{array}$ \\
\hline t lateral, QRS < $120 \mathrm{~ms}$ & $\begin{array}{r}1 \\
2 \\
3 \\
4 \\
5 \\
6 \\
7 \\
8 \\
9 \\
10\end{array}$ & $\begin{array}{l} \pm \\
\pm \\
\pm \\
\pm \\
\pm \\
\pm \\
\pm \\
\pm \\
+ \\
+\end{array}$ & $\begin{array}{l} \pm \\
+ \\
+ \\
+ \\
+ \\
+ \\
\pm \\
+ \\
+ \\
+\end{array}$ & $\begin{array}{l} \pm \\
+ \\
+ \\
+ \\
+ \\
+ \\
\pm \\
+ \\
+ \\
-\end{array}$ & $\begin{array}{l} \pm \\
\pm \\
\pm \\
\pm \\
\pm \\
\pm \\
\pm \\
\pm \\
-\end{array}$ & $\begin{array}{l} \pm \\
- \\
- \\
- \\
- \\
- \\
\pm \\
\pm \\
\pm \\
+\end{array}$ & $\begin{array}{l} \pm \\
+ \\
+ \\
+ \\
+ \\
+ \\
\pm \\
+ \\
+ \\
\pm\end{array}$ & $\begin{array}{l}+ \\
+ \\
\pm \\
+ \\
+ \\
+ \\
+ \\
\pm \\
+ \\
\pm\end{array}$ & $\begin{array}{l}+ \\
+ \\
\pm \\
+ \\
+ \\
+ \\
+ \\
+ \\
+ \\
\pm\end{array}$ & $\begin{array}{l}+ \\
+ \\
\pm \\
+ \\
\pm \\
+ \\
+ \\
\pm \\
+ \\
+\end{array}$ & $\begin{array}{l}+ \\
+ \\
+ \\
+ \\
+ \\
+ \\
+ \\
+ \\
+ \\
+\end{array}$ & $\begin{array}{l} \pm \\
+ \\
+ \\
+ \\
+ \\
+ \\
\pm \\
\pm \\
+ \\
+\end{array}$ & $\begin{array}{l} \pm \\
\pm \\
\pm \\
\pm \\
t \\
+ \\
- \\
\pm \\
+ \\
\pm\end{array}$ & $\begin{array}{l}\mathbf{B} \\
\mathbf{A} \\
\mathbf{B} \\
\mathbf{A} \\
\mathbf{B} \\
\mathbf{A} \\
\mathbf{A} \\
\mathbf{A} \\
\mathbf{A} \\
\mathbf{A}\end{array}$ & $\begin{array}{l}\mathbf{Y} \\
\mathbf{N} \\
\mathbf{N} \\
\mathbf{N} \\
\mathbf{N} \\
\mathbf{N} \\
\mathbf{Y} \\
\mathbf{N} \\
\mathbf{N} \\
\mathbf{N}\end{array}$ & $\begin{array}{l}\mathbf{N} \\
\mathbf{Y} \\
\mathbf{N} \\
\mathbf{Y} \\
\mathbf{N} \\
\mathbf{N} \\
\mathbf{Y} \\
\mathbf{N} \\
\mathbf{N} \\
\mathbf{N}\end{array}$ \\
\hline ft posterior, $\mathrm{QRS}>120 \mathrm{~ms}$ & $\begin{array}{l}1 \\
2 \\
3 \\
4 \\
5 \\
6 \\
7\end{array}$ & $\begin{array}{l} \pm \\
\pm \\
\pm \\
+ \\
+ \\
+ \\
+\end{array}$ & $\begin{array}{l} \pm \\
+ \\
\pm \\
\pm \\
\pm \\
+ \\
+\end{array}$ & $\begin{array}{l} \pm \\
+ \\
\pm \\
\pm \\
- \\
- \\
+\end{array}$ & $\begin{array}{l} \pm \\
\pm \\
\pm \\
- \\
- \\
-\end{array}$ & $\begin{array}{l}+ \\
\pm \\
\pm \\
\pm \\
t \\
+ \\
+\end{array}$ & $\begin{array}{l} \pm \\
t \\
\pm \\
\pm \\
\pm \\
\pm \\
\pm\end{array}$ & $\begin{array}{l}+ \\
+ \\
+ \\
+ \\
+ \\
\pm \\
+\end{array}$ & $\begin{array}{l}+ \\
+ \\
+ \\
+ \\
+ \\
+ \\
+\end{array}$ & $\begin{array}{l}+ \\
+ \\
+ \\
+ \\
+ \\
+ \\
+\end{array}$ & $\begin{array}{l}+ \\
+ \\
+ \\
+ \\
+ \\
+ \\
+\end{array}$ & $\begin{array}{l} \pm \\
t \\
\pm \\
t \\
+ \\
+ \\
+\end{array}$ & $\begin{array}{l} \pm \\
+ \\
- \\
+ \\
+ \\
+ \\
+\end{array}$ & $\begin{array}{l}\mathbf{A} \\
\mathbf{A} \\
\mathbf{A} \\
\mathbf{A} \\
\mathbf{A} \\
\mathbf{A} \\
\mathbf{A}\end{array}$ & $\begin{array}{l}\mathbf{N} \\
\mathbf{N} \\
\mathbf{N} \\
\mathbf{N} \\
\mathbf{N} \\
\mathbf{N} \\
\mathbf{N}\end{array}$ & $\begin{array}{l}\mathbf{N} \\
\mathbf{N} \\
\mathbf{Y} \\
\mathbf{N} \\
\mathbf{Y} \\
\mathbf{N} \\
\mathbf{N}\end{array}$ \\
\hline ft posterior, QRS $<120 \mathrm{~ms}$ & $\begin{array}{l}1 \\
2 \\
3 \\
4 \\
5 \\
6\end{array}$ & $\begin{array}{l}-\overline{ \pm} \\
\pm \\
\pm \\
+ \\
\pm\end{array}$ & $\begin{array}{l}+ \\
+ \\
\pm \\
+ \\
- \\
\pm\end{array}$ & $\begin{array}{l}+ \\
+ \\
\pm \\
\pm \\
\pm \\
\pm\end{array}$ & $\begin{array}{l} \pm \\
\pm \\
\pm \\
\pm \\
\pm\end{array}$ & $\begin{array}{l} \pm \\
\pm \\
\pm \\
\pm \\
\pm \\
\pm\end{array}$ & $\begin{array}{l}+ \\
+ \\
\pm \\
+ \\
- \\
\pm\end{array}$ & $\begin{array}{l}+ \\
+ \\
\pm \\
+ \\
\pm \\
\pm\end{array}$ & $\begin{array}{l}+ \\
+ \\
\pm \\
+ \\
+ \\
\pm\end{array}$ & $\begin{array}{l}+ \\
+ \\
+ \\
+ \\
+ \\
+\end{array}$ & $\begin{array}{l}+ \\
+ \\
+ \\
+ \\
+ \\
+\end{array}$ & $\begin{array}{l} \pm \\
+ \\
+ \\
+ \\
+ \\
+\end{array}$ & $\begin{array}{l} \pm \\
\pm \\
\pm \\
\pm \\
\pm \\
\pm\end{array}$ & $\begin{array}{l}\mathbf{B} \\
\mathbf{A} \\
\mathbf{A} \\
\mathbf{A} \\
\mathbf{A} \\
\mathbf{B}\end{array}$ & $\begin{array}{l}\mathbf{N} \\
\mathbf{N} \\
\mathbf{N} \\
\mathbf{N} \\
\mathbf{Y} \\
\mathbf{N}\end{array}$ & $\begin{array}{l}\mathbf{N} \\
\mathbf{N} \\
\mathbf{N} \\
\mathbf{N} \\
\mathbf{N} \\
\mathbf{N}\end{array}$ \\
\hline
\end{tabular}

, positive delta wave; - , negative delta wave; \pm , isoelectric delta wave.

TPW, Wolff-Parkinson-White syndrome.

I, no (criteria did not identify location of pathway); $Y$, yes (criteria did identify location of pathway).

ble 3 Usefulness of established criteria to locate the accessory pathway by the resting 12 lead electrocardiogram in patients with the olff-Parkinson-White syndrome

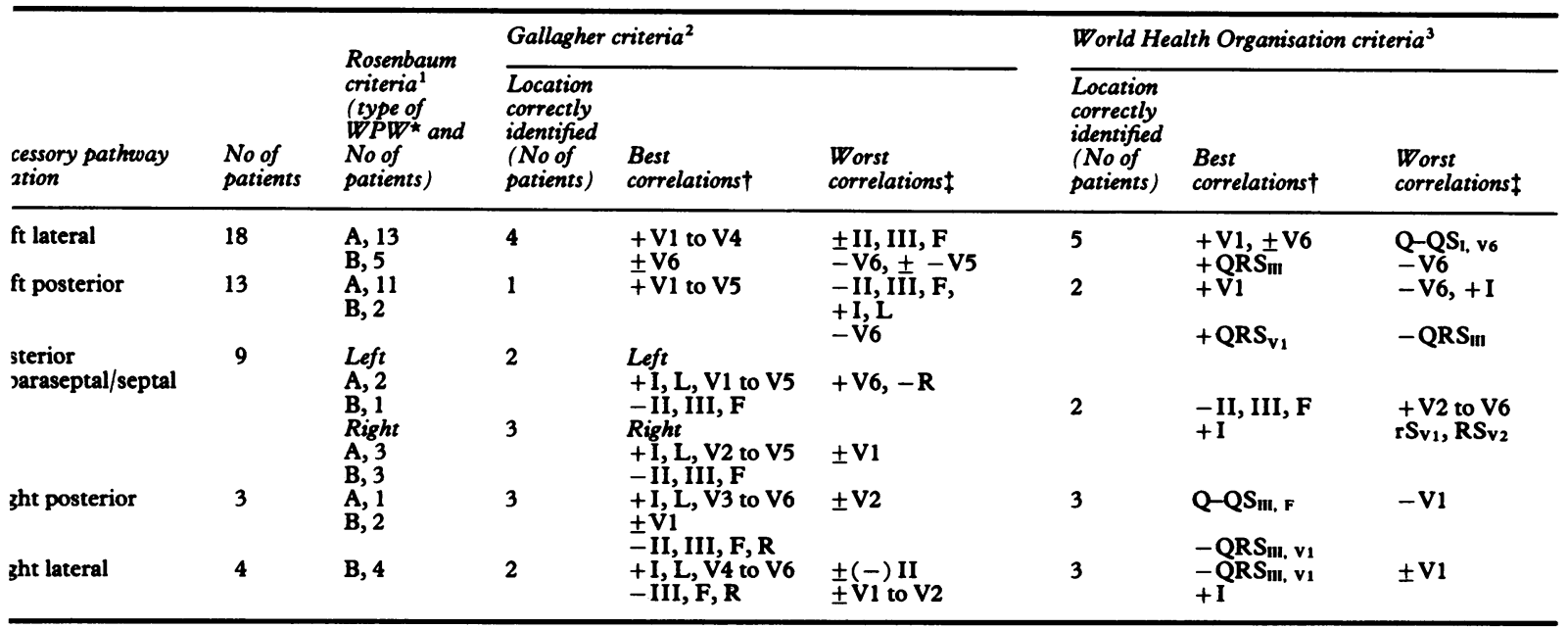

, positive delta wave;

, negative delta wave;

, isoelectric delta wave;

IPW, Wolff-Parkinson-White syndrome.

$: 60 \%$ of electrocardiograms had these findings.

$: 40 \%$ of electrocardiograms had these findings. 
Table 4 Vector direction at 10,20,30, and $40 \mathrm{~ms}$ on ihe resting vectorcardiogram in patients with left lateral or left posterior accessory. pathways

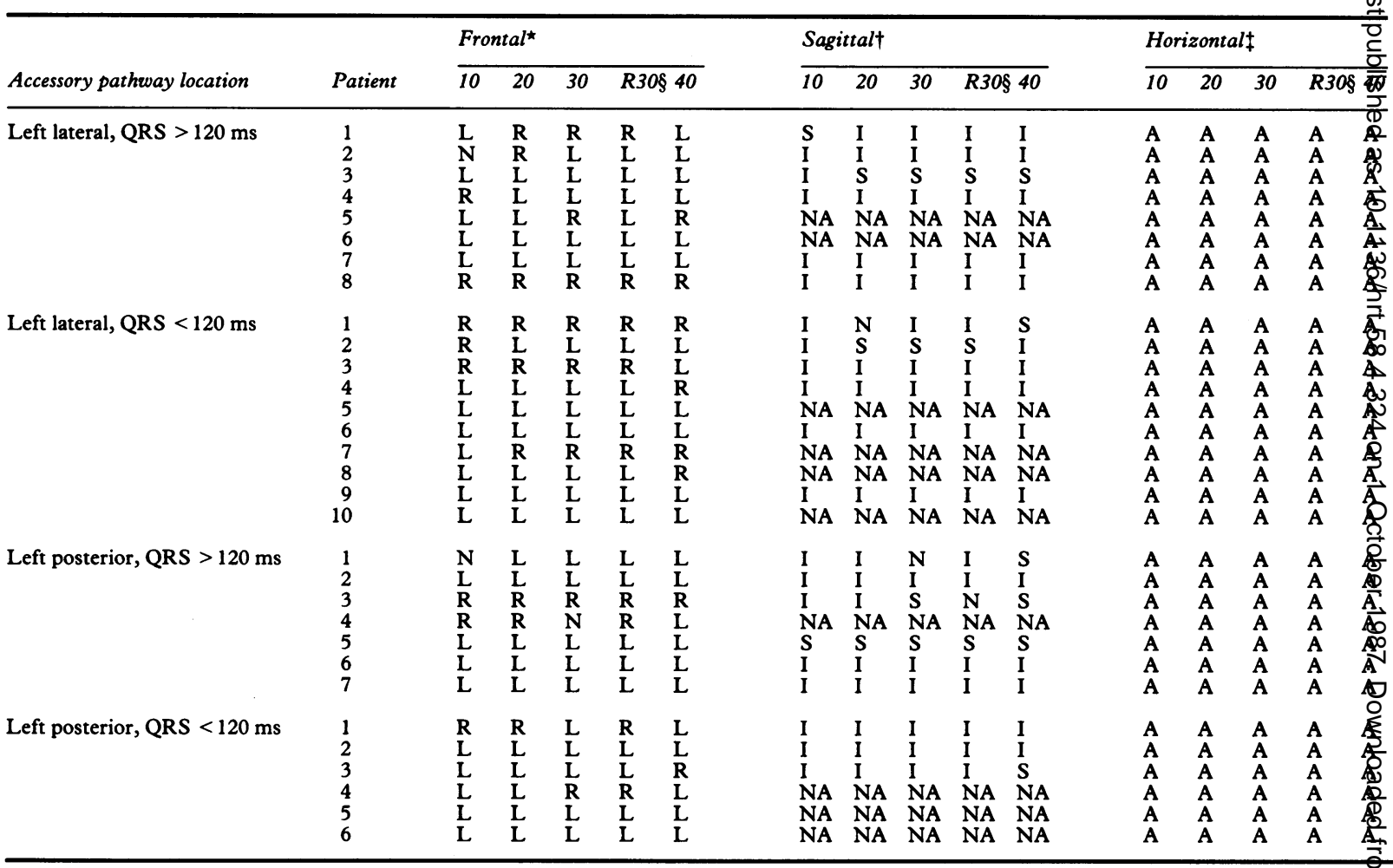

${ }^{\star} L$, left; $R$, right; $N$, neutral. $\nmid I$, inferior; $S$, superior; NA, not available. $\ddagger A$, anterior. $§ R 30$, mean vector of first $30 \mathrm{~ms}$.

Table 5 Delta wave polarity on the resting 12 lead electrocardiogram in patients with posterior paraseptal, right posterior, or right latera accessory pathways

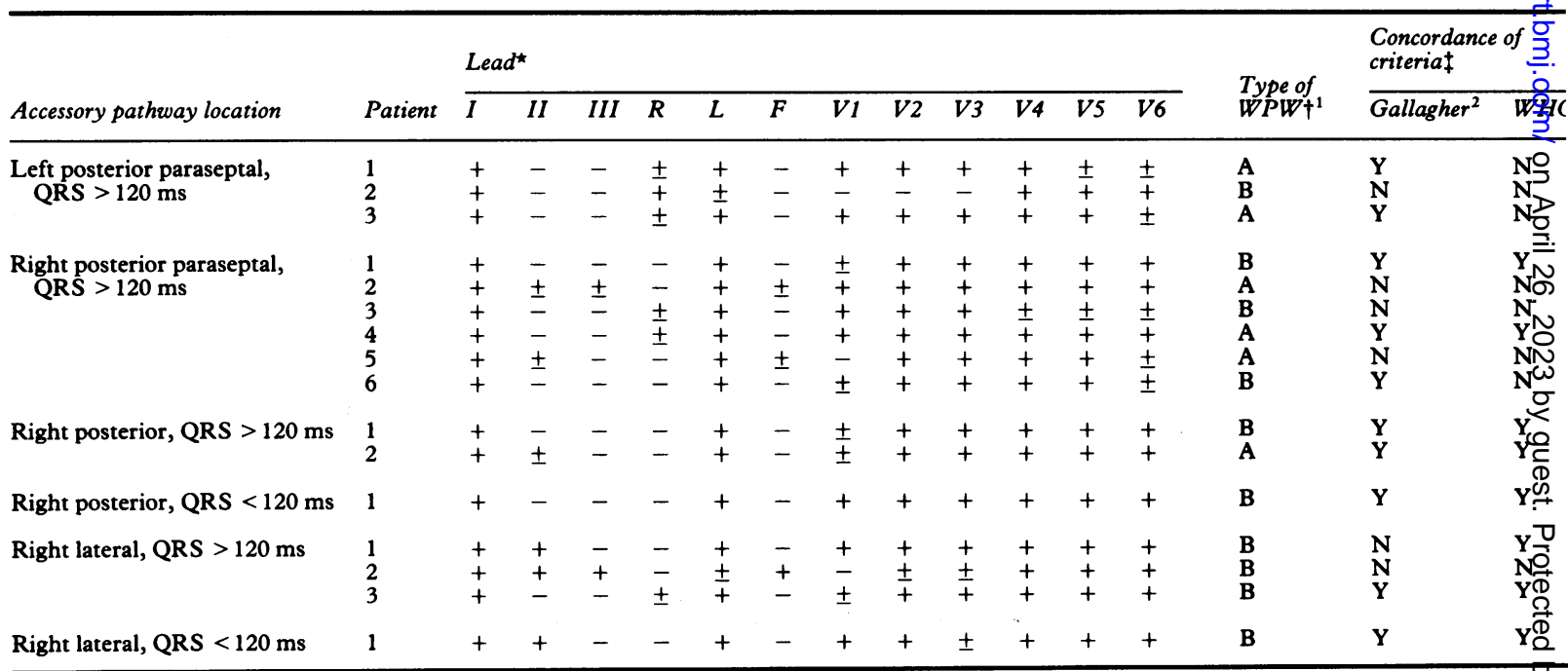

$\star+$, positive delta wave; - , negative delta wave; \pm , isoelectric delta wave.

+WPW, Wolff-Parkinson-White syndrome.

$\ddagger N$, no (criteria did not identify location of pathway); $Y$, yes (criteria did identify location of pathway). 
le 6 Vector direction at 10,20,30, and 40 ms on the resting vectorcardiogram in patients with posterior paraseptal, right posterior, ight lateral accessory pathways

\begin{tabular}{|c|c|c|c|c|c|c|c|c|c|c|c|c|c|c|c|c|}
\hline \multirow[b]{2}{*}{ ssory pathway location } & \multirow[b]{2}{*}{ Patient } & \multicolumn{5}{|c|}{ Frontal } & \multicolumn{5}{|c|}{ Sagittal } & \multicolumn{5}{|c|}{ Horizontal } \\
\hline & & 10 & 20 & 30 & $R 30$ & 40 & 10 & 20 & 30 & $R 30$ & 40 & 10 & 20 & 30 & $R 30$ & 40 \\
\hline $\begin{array}{l}\text { posterior paraseptal, } \\
\text { RS }>120 \mathrm{~ms}\end{array}$ & $\begin{array}{l}1 \\
2 \\
3\end{array}$ & $\begin{array}{l}\text { L } \\
\text { R } \\
\text { L }\end{array}$ & $\begin{array}{l}\text { L } \\
\text { L } \\
\text { L }\end{array}$ & $\begin{array}{l}\text { L } \\
\text { L } \\
\text { L }\end{array}$ & $\begin{array}{l}\text { L } \\
\text { L } \\
\text { L }\end{array}$ & $\begin{array}{l}\text { L } \\
\text { L } \\
\text { L }\end{array}$ & $\begin{array}{l}S \\
\text { I } \\
\text { NA }\end{array}$ & $\begin{array}{l}\text { S } \\
\text { I } \\
\text { NA }\end{array}$ & $\begin{array}{l}S \\
\text { I } \\
\text { NA }\end{array}$ & $\begin{array}{l}\text { S } \\
\text { I } \\
\text { NA }\end{array}$ & $\begin{array}{l}S \\
\text { SA }\end{array}$ & $\begin{array}{l}\mathbf{A} \\
\mathbf{P} \\
\mathbf{A}\end{array}$ & $\begin{array}{l}\mathbf{A} \\
\mathbf{P} \\
\mathbf{A}\end{array}$ & $\begin{array}{l}\mathbf{A} \\
\mathbf{P} \\
\mathbf{A}\end{array}$ & $\begin{array}{l}\mathbf{A} \\
\mathbf{P} \\
\mathbf{A}\end{array}$ & $\begin{array}{l}\mathbf{A} \\
\mathbf{P} \\
\mathbf{A}\end{array}$ \\
\hline $\begin{array}{l}\text { It posterior paraseptal, } \\
\mathrm{RS}>120 \mathrm{~ms}\end{array}$ & $\begin{array}{l}1 \\
2 \\
3 \\
4 \\
5 \\
6\end{array}$ & $\begin{array}{l}\text { L } \\
\text { L } \\
\text { R } \\
\text { L } \\
\text { L } \\
\text { L }\end{array}$ & $\begin{array}{l}\text { L } \\
\text { L } \\
\text { L } \\
\text { L } \\
\text { L } \\
\text { L }\end{array}$ & $\begin{array}{l}\text { L } \\
\text { L } \\
\text { L } \\
\text { L } \\
\text { L } \\
\text { L }\end{array}$ & $\begin{array}{l}\text { L } \\
\text { L } \\
\text { L } \\
\text { L } \\
\text { L } \\
\text { L }\end{array}$ & $\begin{array}{l}\text { L } \\
\text { L } \\
\text { L } \\
\text { L } \\
\text { L } \\
\text { L }\end{array}$ & $\begin{array}{l}\text { I } \\
\text { N } \\
\text { S } \\
\text { NA } \\
\text { NA } \\
\text { I }\end{array}$ & $\begin{array}{l}\mathbf{I} \\
\mathbf{N} \\
\mathbf{S} \\
\mathbf{N A} \\
\text { NA } \\
\mathbf{S}\end{array}$ & $\begin{array}{l}S \\
\text { S } \\
\text { S } \\
\text { NA } \\
\text { NA } \\
\text { S }\end{array}$ & $\begin{array}{l}\text { S } \\
\text { S } \\
\text { S } \\
\text { NA } \\
\text { NA } \\
\text { S }\end{array}$ & $\begin{array}{l}S \\
S \\
\text { S } \\
\text { NA } \\
\text { NA } \\
S\end{array}$ & $\begin{array}{l}\mathbf{A} \\
\mathbf{A} \\
\mathbf{A} \\
\mathbf{A} \\
\mathbf{P} \\
\mathbf{A}\end{array}$ & $\begin{array}{l}\mathbf{A} \\
\mathbf{A} \\
\mathbf{A} \\
\mathbf{A} \\
\mathbf{P} \\
\mathbf{A}\end{array}$ & $\begin{array}{l}\mathbf{A} \\
\mathbf{A} \\
\mathbf{P} \\
\mathbf{P} \\
\mathbf{P} \\
\mathbf{P}\end{array}$ & $\begin{array}{l}\mathbf{A} \\
\mathbf{A} \\
\mathbf{A} \\
\mathbf{A} \\
\mathbf{P} \\
\mathbf{P}\end{array}$ & $\begin{array}{l}\mathbf{A} \\
\mathbf{A} \\
\mathbf{P} \\
\mathbf{A} \\
\mathbf{P} \\
\mathbf{P}\end{array}$ \\
\hline it posterior, $\mathrm{QRS}>120 \mathrm{~ms}$ & $\begin{array}{l}1 \\
2\end{array}$ & $\begin{array}{l}\mathbf{L} \\
\mathbf{L}\end{array}$ & $\begin{array}{l}\mathrm{L} \\
\mathrm{L}\end{array}$ & $\begin{array}{l}\mathrm{L} \\
\mathrm{L}\end{array}$ & $\begin{array}{l}\mathrm{L} \\
\mathrm{L}\end{array}$ & $\begin{array}{l}\mathrm{L} \\
\mathbf{L}\end{array}$ & $\overline{N A}$ & $\overline{\mathbf{N A}}$ & $\begin{array}{l}\text { S } \\
\text { NA }\end{array}$ & $\begin{array}{l}\text { SA } \\
\text { NA }\end{array}$ & $\begin{array}{l}\text { SA } \\
\text { NA }\end{array}$ & $\begin{array}{l}\mathbf{A} \\
\mathbf{P}\end{array}$ & $\stackrel{\mathbf{A}}{\mathbf{N}}$ & $\begin{array}{l}\mathbf{A} \\
\mathbf{A}\end{array}$ & $\begin{array}{l}\mathbf{A} \\
\mathbf{A}\end{array}$ & $\begin{array}{l}\mathbf{P} \\
\mathbf{A}\end{array}$ \\
\hline tt posterior, $Q R S<120 \mathrm{~ms}$ & 1 & $\mathbf{R}$ & $\mathbf{L}$ & $\mathbf{L}$ & $\mathbf{L}$ & L & NA & NA & NA & NA & NA & $\mathbf{A}$ & $\mathbf{A}$ & $\mathbf{A}$ & $\mathbf{A}$ & $\mathbf{A}$ \\
\hline
\end{tabular}

le vector was not obtained for this patient.

eft; $R$, right; I, inferior; S, superior; NA, not available; $N$, neutral; $A$, anterior; $P$, posterior; R30, mean vector of first 30 ms.

lagher criteria an accessory pathway location was correctly identified by the 12 lead electrocardiogram if the polarity of the delta wave in at least nine of the 12 leads conformed with the published criteria (fig 2); and for the World Health Organisation criteria ${ }^{3}$ an accessory pathway location was correctly identified by the 12 lead electrocardiogram if all of the criteria (table 1) except one were correct.

\section{Results}

\section{LEFT LATERAL ACCESSORY PATHWAY}

Type A Wolff-Parkinson-White syndrome was present in 13 of the 18 patients (table 2). The pathway location was correctly identified by the Gallagher criteria ${ }^{2}$ in four of the patients and by the World Health Organisation criteria ${ }^{3}$ in five (table 3 ).

The vector for R30 (table 4) was anterior in all 18 patients, oriented to the left in 13 of the patients, and inferior in 10 of the 12 patients for whom such information was available.

\section{LEFT POSTERIOR ACCESSORY PATHWAY}

Type A Wolff-Parkinson-White syndrome was present in 11 of the 13 patients (table 2). The pathway location was correctly identified by the Gallagher criteria ${ }^{2}$ in one patient and by the World Health Organisation criteria ${ }^{3}$ in two (table 3 ).

The vector for R30 (table 4) was anterior in all patients, oriented to the left in nine of the 13 patients, and inferior in seven of the nine patients for whom such information was available.
POSTERIOR PARASEPTAL ACCESSORY PATHWAY (TABLE 5)

Type A Wolff-Parkinson-White syndrome was present in two of the three patients with a left posterior paraseptal accessory pathway and three of the six with a right posterior paraseptal accessory pathway. The pathway location was correctly identified by the Gallagher criteria ${ }^{2}$ in five of the nine patients and by the World Health Organisation criteria ${ }^{3}$ in two (table 3).

The vector for R30 (table 6) was anterior in six of the nine patients, oriented to the left in all nine patients, and superior in five of the six patients for whom such information was available.

\section{RIGHT POSTERIOR ACCESSORY PATHWAY (TABLE 5)}

Type B Wolff-Parkinson-White syndrome was present in two of the three patients. All three patients had the pathway location correctly identified by the Gallagher criteria ${ }^{2}$ and the World Health Organisation criteria ${ }^{3}$ (table 3 ).

The vector for R30 (table 6) was anterior in all three patients, oriented to the left in all three patients, and superior in the one patient for whom such information was available.

\section{RIGHT LATERAL ACCESSORY PATHWAY (TABLE 5)}

Type B Wolff-Parkinson-White syndrome was present in all four patients. The pathway location was correctly identified by the Gallagher criteria ${ }^{2}$ in 
two of the four patients and by the World Health Organisation criteria ${ }^{3}$ in three (table 3 ).

The vector for R30 (table 6) was anterior in two of the patients, oriented to the left in two of the patients, and superior in two of the patients. A vector was not obtained in the fourth patient.

\section{Discussion}

The concept that pre-excitation in WolffParkinson-White syndrome was the result of conduction over an accessory atrioventricular pathway remained speculative until 1967 when Durrer and Roos provided evidence for atrioventricular conduction over an accessory pathway by epicardial mapping of the heart. ${ }^{11}$ Work by Burchell et al ${ }^{12}$ and Boineau and Moore ${ }^{13}$ further substantiated this belief, and in 1968, Cobb et $a l^{14}$ and Sealy et $a l^{15}$ were the first to divide an accessory pathway successfully. Because the Wolff-Parkinson-White syndrome can be cured by surgical ablation the ability to locate the site of pre-excitation has become of great importance.

Electrocardiography and vectorcardiography ${ }^{16-18}$ have remained the two most widely used methods to predict the location of the accessory pathway. Recently, echocardiography, ${ }^{19}$ body surface mapping, ${ }^{2021}$ phase imaging, ${ }^{22}$ and the moving dipole ${ }^{23}$ have also been used in an attempt to locate the site of an accessory pathway.

TYPE A AND TYPE B WOLFF-PARKINSON-WHITE SYNDROME

The classification by Rosenbaum et $a l^{1}$ of Wolff-Parkinson-White syndrome into type $A$ or type $B$ has stood the test of time because of its simplicity. Type A was believed to be due to left posterobasal pre-excitation, and type $B$ to right lateral pre-excitation. ${ }^{24}$ The Rosenbaum criteria correctly identified a left-sided accessory pathway in $76 \%$ of our patients and a right-sided accessory pathway in $69 \%$ of our patients. This classification, however, does not help to separate free wall and paraseptal/septal locations.

\section{VECTORCARDIOGRAPHY IN}

WOLFF-PARKINSON-WHITE SYNDROME

Vectorcardiography has also been used to locate the accessory pathway. In this study it did not accurately identify the location of the accessory pathway because $(a)$ the vector was anterior in most $(89 \%)$ of the patients; $(b)$ the vector was to the left in $71 \%$ of the patients with a left free wall accessory pathway, in all patients with a posterior paraseptal accessory pathway, and in $83 \%$ of the patients with a right posterior or right lateral accessory pathway; and (c) the vector was inferior in $81 \%$ of the patients with a left free wall accessory pathway, superior in $83 \%$ of the patients with a posterior paraseptal accessory pathway, and superior in $75 \%$ of the patients with a right free wall accessory pathway.

These findings do not support a previous study that suggested a vector directed anteriorly and to the right indicated a left lateral accessory pathway and a vector directed to the left and anteriorly indicated a paraseptal accessory pathway. ${ }^{25}$ The suggestion in another study that a left lateral accessory pathway had a $10 \mathrm{~ms}$ vector directed inferiorly and a $20 \mathrm{~ms}$ vector directed to the right ${ }^{26}$ was not confirmed by our study.

\section{ELECTROCARDIOGRAPHY AND EPICARDIAL} MAPPING CORRELATIONS

Epicardial mapping during operation provides an opportunity for a comparison with the results of the non-invasive determination of accessory pathway location. Boineau et al, Frank, and Gallagher et al, using 12 lead electrocardiography, ${ }^{5728}$ and Tonkin et al, using vectorcardiography, ${ }^{26}$ provided the first correlations. However, criteria for locating the accessory pathway from the 12 lead electrocardiogram based on the delta wave polarity were not adopted until the important article by Gallagher et $a l .{ }^{2}$ Their review provided information relating the delta wave polarity in the 12 standard leads obtained during rapid atrial pacing (resulting in maximal preexcitation) to the accessory pathway location determined by epicardial mapping during operation for 10 different accessory pathway locations. They suggested that their results should be applied to patients in whom the QRS complexes were more than $140 \mathrm{~ms}$.

Recently, the World Health Organisation ${ }^{3}$ developed a classification of accessory pathway location according to the 12 lead electrocardiogram from a composite of previous studies ${ }^{12529}$ and suggested six sets of criteria for accessory pathway location. Because of fusion caused by conduction over the atrioventricular node and the accessory pathway, they found that pre-excitation and the width of the QRS complex may vary but they did not strictly limit the classification to a certain QRS width. From a practical standpoint, patients with the Wolff-Parkinson-White syndrome who clearly have pre-excitation on the resting 12 lead electrocardiogram often have a QRS width of less than $120 \mathrm{~ms}$. In our series, 18 of the 47 patients had a QRS width of $\stackrel{?}{?}$ less than $120 \mathrm{~ms}$ and only 10 had a QRS of $140 \mathrm{~ms}$ or more.

The previously reported criteria ${ }^{2} 3$ were developed and are useful for identifying the accessory pathway location from an electrocardiogram 
obtained during maximal pre-excitation, which often requires atrial pacing. Our study shows that the previously reported criteria for accessory pathway location cannot be applied accurately to the resting 12 lead electrocardiogram. Only $32 \%$ of the patients in our series showed concordance with the Gallagher and World Health Organisation criteria. Both sets of criteria were inaccurate for left free wall and posterior paraseptal pathway locations. The results obtained in patients with a QRS width of more than $120 \mathrm{~ms}$ were not different from those in patients with a width of less than $120 \mathrm{~ms}$.

The results in our study differ from previous criteria ${ }^{23}$ as follows: (a) a negative delta wave or a $Q$ wave in V6 rarely occurs and should not be considered necessary for locating a left lateral or left posterior accessory pathway ${ }^{232728}$; (b) inferior leads II, III, and aVF often have a positive delta wave in the left lateral and left posterior accessory pathway ${ }^{2728}$; (c) a negative QRS complex in leads V1 and III does not permit right lateral and right posterior accessory pathways to be discriminated; and (c) the combination of a positive QRS complex in lead V1 and a negative QRS complex in lead III rarely occurs with a left posterior accessory pathway.

The limitations of our study are as follows: $(a)$ in 15 of the 47 patients the delta wave was not analysed simultaneously in any lead, but great care was taken to analyse the first $40 \mathrm{~ms}$ of ventricular activation in each lead; and (b) the number of right-sided accessory pathways was small and no patients with anterior septal pathways were evaluated.

\section{Clinical implications}

The resting 12 lead electrocardiogram is not helpful for identifying the precise location of an accessory pathway by published criteria. If such information is required, detailed electrophysiological testing should be performed.

\section{References}

1 Rosenbaum FF, Hecht HH, Wilson FN, Johnston FD. The potential variations of the thorax and the esophagus in anomalous atrioventricular excitation (Wolff-Parkinson-White syndrome). Am Heart $J$ 1945;29:281-326.

2 Gallagher JJ, Pritchett ELC, Sealy WC, Kasell J, Wallace AG. The preexcitation syndromes. Prog Cardiovasc Dis 1978;20:285-327.

3 Willems JL, Robles de Medina EO, Bernard R, et al. Criteria for intraventricular conduction disturbances and pre-excitation. J Am Coll Cardiol 1985;5: 1261-75.

4 Wolff L, Parkinson J, White PD. Bundle-branch block with short $P-R$ interval in healthy young people prone to paroxysmal tachycardia. Am Heart $J$ 1930;5:685-704.

5 Boineau JP, Moore EN, Spear JF, Sealy WC. Basis of static and dynamic electrocardiographic variations in Wolff-Parkinson-White syndrome: anatomic and electrophysiologic observations in right and left ventricular preexcitation. Am J Cardiol 1973;32:32-45.

6 Hammill SC, Pritchett ELC, Klein GJ, Smith WM, Gallagher JJ. Accessory atrioventricular pathways that conduct only in the antegrade direction. Circulation 1980;62:1335-40.

7 Hammill SC, Holmes DR Jr, Wood DL, et al. Electrophysiologic testing in the upright position: improved evaluation of patients with rhythm disturbances using a tilt table. J Am Coll Cardiol 1984;4:65-71.

8 Holmes DR Jr, Danielson GK, Gersh BJ, et al. Surgical treatment of accessory atrioventricular pathways and symptomatic tachycardia in children and young adults. Am J Cardiol 1985;55:1509-12.

9 Gallagher JJ, Pritchett ELC, Benditt DG, et al. New catheter techniques for analysis of the sequence of retrograde atrial activation in man. Eur $J$ Cardiol 1977;6:1-14.

10 Holmes DR Jr, Osborn MJ, Gersh B, Maloney JD, Danielson GK. The Wolff-Parkinson-White syndrome: a surgical approach. Mayo Clin Proc 1982;57:345-50.

11 Durrer D, Roos JP. Epicardial excitation of the ventricles in a patient with Wolff-Parkinson-White syndrome (type B). Circulation 1967;35:15-21.

12 Burchell HB, Frye RL, Anderson MW, McGoon DC. Atrioventricular and ventriculoatrial excitation in Wolff-Parkinson-White syndrome (type B): temporary ablation at surgery. Circulation 1967;36:663-72.

13 Boineau JP, Moore EN. Evidence for propagation of activation across an accessory atrioventricular connection in types A and B pre-excitation. Circulation 1970;41:375-97.

14 Cobb FR, Blumenschein SD, Sealy WC, Boineau JP, Wagner GS, Wallace AG. Successful surgical interruption of the bundle of Kent in a patient with Wolff-Parkinson-White syndrome. Circulation 1968;38:1018-29.

15 Sealy WC, Hattler BG Jr, Blumenschein SD, Cobb FR. Surgical treatment of Wolff-Parkinson-White syndrome. Ann Thorac Surg 1969;8:1-10.

16 Bleifer S, Kahn M, Grishman A, Donoso E. Wolff-Parkinson-White syndrome: a vector cardiographic, electrocardiographic and clinical study. Am J Cardiol 1959;4:321-33.

17 Tranchesi J, Guimarães AC, Teixeira V, Pileggi F. Vectorial interpretation of the ventricular complex in Wolff-Parkinson-White syndrome. Am J Cardiol 1959;4:334-40.

18 Lowe KG, Emslie-Smith D, Ward C, Watson H. Classification of ventricular pre-excitation: vectorcardiographic study. Br Heart J 1975;37:9-19.

19 Windle JR, Armstrong WF, Feigenbaum H, Miles WM, Prystowsky EN. Determination of the earliest site of ventricular activation in Wolff-ParkinsonWhite syndrome: application of digital continuous loop two-dimensional echocardiography. J Am Coll 
Cardiol 1986;7:1286-94.

20 Benson DW Jr, Sterba R, Gallagher JJ, Walston A II, Spach MS. Localization of the site of ventricular preexcitation with body surface maps in patients with Wolff-Parkinson-White syndrome. Circulation 1982; 65:1259-68.

21 Groenewegen AS, Spekhorst HHM, Reek EJ. A quantitative method for the localization of the ventricular pre-excitation area in the Wolff-Parkinson-White syndrome using singular value decomposition of body surface potentials. J Electrocardiol 1985; 18:157-67.

22 Rakovec P, Kranjec I, Fettich J, et al. Localization of accessory pathways in Wolff-Parkinson-White syndrome by phase imaging. Cardiology 1983;70: 138-44.

23 Gulrajani RM, Pham-Huy H, Nadeau RA, et al. Application of the single moving dipole inverse solution to the study of the Wolff-Parkinson-White syndrome in man. J Electrocardiol 1984;17:271-87.

24 Giraud G, Latour H, Puech P, Roujon J. Les troubles de rythme du syndrome de Wolff-Parkinson-White. Analyse électrocardiographique endocavitaire. Arch Mal Coeur 1956;49:101-33.

25 Talwar KK, Blomström P, Edvardsson N, William-
Olsson G, Olsson SB. Spatial vectorcardiography in the Wolff-Parkinson-White syndrome: correlation with epicardial mapping findings. $P A C E$ 1984; 7:979-84.

26 Tonkin AM, Wagner GS, Gallagher JJ, Cope GD, Kasell J, Wallace AG. Initial forces of ventricular depolarization in the Wolff-Parkinson-White syndrome: analysis based upon localization of the accessory pathway by epicardial mapping. Circulation 1975;52:1030-6.

27 Frank R. Apport des investigations endocavitaires et de cartographies epicardiques dans l'étude des syndromes de pré-excitation ventriculaire. Thése pour le Doctorat de Medicine: Paris, Editions Medicals et Universitaires, 1974.

28 Gallagher JJ, Svenson RH, Sealy WC, Wallace AG. The Wolff-Parkinson-White syndrome and the preexcitation dysrhythmias: medical and surgical management. Med Clin North Am 1976; 60:101-23.

29 Wellens HJJ, Durrer D. The role of an accessory atrioventricular pathway in reciprocal tachycardia: observations in patients with and without the Wolff-Parkinson-White syndrome. Circulation 1975;52:58-72. 